\title{
Markets and contracts ${ }^{1}$
}

\author{
A. Bisin ${ }^{2}$ J. D. Geanakoplos ${ }^{3} \quad$ P. Gottardi ${ }^{4}$ \\ E. Minelli ${ }^{5} \quad$ H. Polemarchakis ${ }^{6}$
}

December 9, 2010

\footnotetext{
${ }^{1}$ We wish to thank Laura Carosi, Tito Pietra and two referees. Bisin acknowledges financial support from NSF grant SES-9818844.

${ }^{2}$ Department of Economics, New York University; alberto.bisin@econ.nyu.edu

${ }^{3}$ Cowles Foundation, Yale University;

john.geanakoplos@yale.edu

${ }^{4}$ Department of Economics, European University Institute; piero.gottardi@eui.eu

${ }^{5}$ Dipartimento di Scienze Economiche, Università di Brescia; minelli@eco.unibs.it

${ }^{6}$ Department of Economics, University of Warwick; h.polemarchakis@warwick.ac.uk
} 


\begin{abstract}
Economies with asymmetric information are encompassed by an extension of the model of general competitive equilibrium that does not require an explicit modeling of private information. Sellers have discretion over deliveries on contracts; this is in common with economies with default, incomplete contracts or price rigidities. Competitive equilibria exist and anonymous markets are viable. But, for a generic economy, aa competitive equilibrium allocations are constrained suboptimal: there exist Pareto improving interventions via linear, anonymous taxes.
\end{abstract}

Key words: asymmetric information, competitive markets, equilibrium.

JEL classification numbers: D50, D52, D82. 


\section{Introduction}

Asymmetric information plays an important role in the allocation of resources. The implications of moral hazard and adverse selection have been analyzed extensively from a game theoretic perspective, which has clarified the nature and implications of principal-agent relations, signaling, and contracts. This analysis has been, mostly, confined, however, to situations that involve a small number of individuals, which both limits its relevance and deprives it of the advantages of anonymous trade.

The model of general competitive equilibrium allows, even requires, that individuals be many; it imposes no restrictions on their heterogeneity; it limits the information of individuals to their characteristics and not those of others; and it postulates, even explains that their behavior, deriving from their rationality, is not strategic: it describes competitive markets.

We argue here that economies with asymmetric information are encompassed by an extension of the model of general competitive equilibrium that does not require an explicit modeling of private information. The modifications include, in the description of the economy,

- the exchange of commodities indirectly, through the exchange of contracts that pay off in multiple commodities,

- the ability of individuals to exercise discretion on the deliveries on contracts, which derives from their private information, and

- the access of individuals to technologies that transform their endowments prior to trade,

and, in the definition of equilibrium,

- the pooling of the deliveries of sellers and their distribution to buyers in proportion to their purchases.

The main feature of models of asymmetric information, whatever the specific nature of informational asymmetries, including both moral hazard and adverse selection, is the discretion of sellers over deliveries on contracts. The pooling of payoffs guarantees equilibrium in the market for commodities when prices attain equilibrium in the market for contracts; this was noted first in Dubey, Geanakoplos and Shubik (1990, 2005).

Deliveries on contracts remain under private control, while trades in contracts take place in large anonymous markets and hence individual trades are also only privately observable. The appropriate notion of constrained efficiency restricts therefore interventions to lump-sum transfers, common to all agents, and the linear taxation of trades in contracts. Indeed, we show that for a generic economy equilibria are not constrained efficient; this confirms an insight by Greenwald and Stiglitz (1986).

An alternative approach to markets with asymmetric information was pioneered by Prescott and Townsend (1984), ${ }^{1}$ who studied the implementation

\footnotetext{
${ }^{1}$ Also, Kehoe, Levine and Prescott (2002) and Kocherlakota (1998).
} 
of incentive-constrained efficient allocations as decentralized equilibria. To this effect, individual trades must be observable and appropriate restrictions on individually feasible trades must be added in the equilibrium notion. Without these restrictions, equilibria are not in general incentive-constrained efficient ${ }^{2}$. This is in line with our result, which focuses on the polar case of large, anonymous markets, where individual trades are not observable.

\section{The economy and equilibrium}

Individuals are $i \in \mathcal{I}=\{1, \ldots, I\}$.

Commodities, are $l \in \mathcal{L}=\{1, \ldots, L\}$; a bundle of commodities is ${ }^{3} x=$ $\left(\ldots, x_{l}, \ldots\right)^{\prime}$.

Commodities are exchanged indirectly, through the exchange of contracts.

An individual is described by his consumption set, $\mathcal{X}^{i}$, a set of bundles of commodities, his utility function over consumption bundles, $u^{i}$, with domain the consumption set, and by his endowment, $e^{i}$, a bundle of commodities.

Contracts are $m \in \mathcal{M}=\{1, \ldots, M\}$.

A portfolio of contracts sold, short positions in the market for contracts, is $\phi=\left(\ldots, \phi_{m}, \ldots\right)^{\prime} \geq 0$; a portfolio of contracts purchased, long positions in the market for contracts, is $\theta=\left(\ldots, \theta_{m}, \ldots\right)^{\prime} \geq 0$. The sale of $\phi_{m}$ units of contract $m$ constitutes the obligation to deliver a bundle of commodities, $\phi_{m} d_{m}=\left(\ldots, \phi_{m} d_{l, m}, \ldots\right)^{\prime}$, where $d_{m}=\left(\ldots, d_{l, m}, \ldots\right)^{\prime} \geq 0$ is also chosen by the seller. On the other hand, the purchase of $\theta_{m}$ units of the contract constitutes the right to receive the bundle of commodities $\theta_{m} r_{m}=\left(\ldots, \theta_{m} r_{l, m}, \ldots\right)^{\prime}$, where $r_{m}=\left(\ldots, r_{l, m}, \ldots\right)^{\prime} \geq 0$ equals the average deliveries made by sellers on the contract.

The specification of each contract is thus given by a pair:

- $\mathcal{D}_{m} \subset \mathcal{R}_{+}^{L}$, that describes the restrictions on the per unit admissible deliveries on the contract: $d_{m} \in \mathcal{D}_{m}$, and

- $\Phi_{m} \subset[0, \infty)$, describing the restrictions on admissible sales of the contract: $\phi_{m} \in \Phi_{m}$.

The payoff, $r_{m}$, to buyers of the contract is then endogenously determined at equilibrium by the average deliveries made by sellers within the set $\mathcal{D}_{m}$.

The set $\mathcal{D}_{m}$ of deliveries on a contract need not be a singleton: the contract need not specify exactly the delivery of a seller; this gives individuals discretion on the bundle of commodities to deliver. Private information over the characteristics of commodities delivered or, more generally, restricted contractual enforceability are encompassed by appropriate specifications of the set of deliveries on the contract. For example, a set of deliveries of the form $\mathcal{D}_{m}=\left\{d_{m}: d_{1, m}+d_{2, m}=1, d_{l, m}=0, l \neq 1,2\right\}$ prescribes the delivery of

\footnotetext{
${ }^{2}$ Hammond (1987, 1989), Cole and Kocherlakota (2001), Citanna and Villanacci (2002), Golosov and Tsyvinski (2007), Panaccione (2007), Fahri, Golosov and Tsyvinski (2009).

3 "'" denotes the transpose.
} 
one unit of a generic commodity (e.g. corn), which can be satisfied by delivering any combination of quantities of commodities 1 or 2 (e.g. two different qualities of corn) that sum up to 1, at the discretion of the seller. Symmetric information and full contractual enforceability correspond to the case where the set of deliveries on each contract is a singleton.

The set $\Phi_{m}$ of sales of the contract need not coincide with the non -negative real line: it need not allow arbitrary sales of the contract. If the set of sales of the contract does coincide with the non-negative real line, individuals face no constraints in the purchase of the contract and pricing is linear. Alternatively, non-linearities in the pricing of a contract are encompassed by the specification of different contracts, $m_{k}: k=1, \ldots$, with identical sets of deliveries, $\mathcal{D}_{m_{k}}=$ $\mathcal{D}_{m_{1}}$, but different sets of restrictions on sales, $\Phi_{m_{k}}$ : arbitrary non-linear prices can be encompassed with each $\Phi_{m_{k}}$ identifying a range of transactions over which pricing is linear.

Aggregate deliveries on each contract are pooled and distributed as payoff to buyers in proportion to their purchases of the contract, thus determining the per unit payoff of the contract, exogenous for buyers:

$$
r_{m} \sum_{i \in \mathcal{I}} \theta_{m}^{i}-\sum_{i \in \mathcal{I}} d_{m}^{i} \phi_{m}^{i}=0, \quad m \in \mathcal{M}
$$

Restrictions on the sales and deliveries on a contract, $\left(d_{m}, \phi_{m}\right) \in \mathcal{D}_{m} \times \Phi_{m}$, are described independently of the characteristics of other contracts, and they do not vary with the individual who sells the contract; this permits anonymity and decentralization in the exchange of contracts. Joint restrictions on the deliveries on a contract and the sales on the contract are encompassed by enlarging the set of contracts.

Across contracts, the set of admissible sales of portfolios of contracts is $\Phi=$ $\times_{m \in \mathcal{M}} \Phi_{m}$; the set of admissible deliveries on contracts sold is $\mathcal{D}=\times_{m \in \mathcal{M}} \mathcal{D}_{m}$.

For a commodity, the deliveries on contracts are $D_{l}=\left(\ldots, d_{l, m}, \ldots\right)$; across contracts and commodities, the deliveries on contracts are

$$
D=\left(\ldots, d_{m}, \ldots\right)=\left(\ldots, D_{l}, \ldots\right)^{\prime} .
$$

For a commodity, the payoffs of contracts are $R_{l}=\left(\ldots, r_{l, m}, \ldots\right)$; across contracts and commodities, payoffs of contracts are

$$
R=\left(\ldots, r_{m}, \ldots\right)=\left(\ldots, r_{l}, \ldots\right)^{\prime} .
$$

A commodity, $l^{*}$, can be traded directly if there exists a contract, $m^{*}=l^{*}$, with deliveries given by the singleton set consisting of the unit vector $\mathbf{1}_{l^{*}}^{L}$ and sales that coincide with the non- negative real line. If a commodity is traded directly, one does not distinguish between the contract and the commodity.

Commodities $l \in \tilde{\mathcal{L}} \subset \mathcal{L}$ are not subject to resale; for these commodities deliveries can only be made out of individuals' initial endowments. This is appropriate for commodities whose characteristics are private information of individuals endowed with these commodities. Even though the set of deliveries 
on a contract is specified independently of the individual who delivers on the contract, for commodities $l \in \tilde{\mathcal{L}} \subset \mathcal{L}$ not subject to resale, the endowment of an individual may effectively reduce the set of admissible deliveries. On the other hand, for the commodities $l \in \mathcal{L} \backslash \tilde{\mathcal{L}}$ agents can make deliveries also out of the bundles obtained as payoff of contracts traded and hence can exercise greater discretion on the delivery on contracts.

An action by an individual, at payoffs of contracts $R$, is

$$
a^{i}=\left(x^{i}, \theta^{i}, \phi^{i}, D^{i} \phi^{i}\right) \in \mathcal{A}^{i}(R)
$$

where the domain of actions of the individual at payoffs of contracts $R$ is

$$
\mathcal{A}^{i}(R)=\left\{\begin{array}{l}
x^{i}=e^{i}+R \theta^{i}-D^{i} \phi^{i} \in \mathcal{X}^{i}, \\
a^{i}: \quad\left(\phi^{i}, D^{i}\right) \in \Phi \times \mathcal{D}, \\
e_{l}^{i}-D_{l}^{i} \phi^{i} \geq 0, \quad l \in \tilde{\mathcal{L}}, \\
\theta^{i} \geq 0 .
\end{array}\right\} .
$$

Prices of contracts are $q=\left(\ldots, q_{m}, \ldots\right)$.

At payoffs of contracts purchased $R$ and prices of contracts $q$, the budget set of an individual is

$$
\beta^{i}(q, R)=\left\{(x, \theta, \phi, D \phi) \in \mathcal{A}^{i}(R): q(\theta-\phi) \leq 0\right\} .
$$

The optimization problem of an individual is

$$
\begin{aligned}
\max & u^{i}(x) \\
\text { s.t } & (x, \theta, \phi, D \phi) \in \beta^{i}(q, R) .
\end{aligned}
$$

Across individuals, a profile of actions is $a=\left(\ldots, a^{i}, \ldots\right)$.

A state of the economy is $(R, a)$, payoffs of contracts and a profile of actions; it is feasible if

$$
r_{m} \sum_{i \in \mathcal{I}} \theta_{m}^{i}-\sum_{i \in \mathcal{I}} d_{m}^{i} \phi_{m}^{i}=0, \quad m \in \mathcal{M}
$$

A weaker feasibility condition requires that

$$
R \sum_{i \in \mathcal{I}} \theta^{i}-\sum_{i \in \mathcal{I}} D^{i} \phi^{i}=0 .
$$

At a feasible state of the economy, markets for commodities clear for each contract, as aggregate deliveries are pooled and distributed as payoff for each contract. The weaker feasibility condition allows deliveries to be pooled across contracts. 
Assumption 1. For every individual, the consumption set is the set of nonnegative bundles of commodities: $\mathcal{X}^{i}=\{x: x \geq 0\}$; the utility function, $u^{i}$, is continuous and non-decreasing; the endowment is non-negative $: e^{i} \geq 0$.

This is standard.

Assumption 2. For every individual, the utility function is strictly monotonically increasing in commodity $l^{*}: u^{i}\left(x+k \mathbf{1}_{l^{*}}^{L}\right)>u^{i}(x)$, for all $x \in \mathcal{X}^{i}$ and all $k>0$; the endowment is strictly positive in commodity $l^{*}: e_{l^{*}}^{i}>0$; and commodity $l^{*}$ is traded directly: the per unit delivery on contract $m^{*}=l^{*} \in \mathcal{M}$ is one unit of commodity $l^{*}$ and 0 of every other commodity: $\mathcal{D}_{m^{*}}=\left\{\mathbf{1}_{l^{*}}^{L}\right\}$, and sales of the contract $m^{*}$ are not restricted: $\Phi_{m^{*}}=[0, \infty)$.

This eliminates local satiation and minimum wealth points: the per unit payoffs of the contract $m^{*}$ sold or purchased coincide, while the utility functions of individuals are strictly monotonically increasing, and the endowments of individuals are positive in the payoff of the contract; the price of the contract is positive, and, for no individual is the endowment a minimum wealth point. More generally, we could specify contract $m^{*}$ as paying off in many commodities.

Commodity $l^{*}$ can be interpreted as a consumption commodity available at contracting, which is exchanged directly; strict monotonicity of the utility function and positivity of the endowment in commodity $l^{*}$ are then natural.

Assumption 3. For every contract, $0 \in \Phi_{m}$; moreover, there exists an individual who can sell the contract: $e^{i} \geq d_{m} \phi_{m}$, for some $\left(d_{m}, \phi_{m}\right) \in \mathcal{D}_{m} \times \Phi_{m}$, with $\phi_{m}>0$.

This guarantees that the budget set of every individual is non-empty, and that every contract is, effectively, in positive aggregate supply.

Assumption 4. For every contract $m \in \mathcal{M} \backslash\left\{m^{*}\right\}$, both the set of deliveries on the contract, $\mathcal{D}_{m}$, and the set of sales of the contract, $\Phi_{m}$, are compact.

Compactness ensures that unbounded arbitrage opportunities do not arise; with deliveries partly at the discretion of the sellers, there may be arbitrage opportunities at all prices.

In a convex economy, for every individual,

1. the utility function, $u^{i}$, is quasi-concave, and

2. the set of per unit deliveries on the contract, $\mathcal{D}_{m}$, and the sets of sales of the contract, $\Phi_{m}$, are convex.

An economy is

$$
\mathcal{E}=\left\{\mathcal{I}, \mathcal{L}, \tilde{\mathcal{L}}, \mathcal{M},\left(\mathcal{X}^{i}, u^{i}, e^{i}\right): i \in \mathcal{I},\left(\mathcal{D}_{m}, \Phi_{m}\right): m \in \mathcal{M}\right\} .
$$

The model described above is the leading model; it encompasses both the standard competitive equilibrium models as well as economies with private information, default or price rigidities. 
Definition 1. A competitive equilibrium is $\left(q^{*}, R^{*}, a^{*}\right)$, prices of contracts and a state of the economy, such that

1. for every individual, the action $a^{i *}=\left(x^{i *}, \theta^{i *}, \phi^{i *}, D^{i *} \phi^{i *}\right)$ is a solution to the optimization problem at prices and payoffs of contracts $\left(q^{*}, R^{*}\right)$,

2. the payoffs of contracts lie in the set of deliveries of contracts: $R^{*} \in \mathcal{D}$,

3. the state of the economy $\left(R^{*}, a^{*}\right)$ is feasible, and

4. the market for contracts clears: $\sum_{i \in \mathcal{I}} \theta^{i *}-\sum_{i \in \mathcal{I}} \phi^{i *}=0$.

If all commodities are traded directly, this is the definition of a competitive equilibrium of Arrow and Debreu (1954) and McKenzie (1954), for an exchange economy.

If the sets of deliveries on all contracts are singletons, while no sales restrictions are operative, this is the definition of a competitive equilibrium with an incomplete asset market as in Radner (1972).

The feasibility condition (3) only restricts the payoff to buyers for traded assets; condition (2) then imposes the rather mild requirement that payoffs of non-traded assets lie in the set of admissible deliveries on contracts, which eliminates trivial no-trade equilibria, as long as $0 \notin \mathcal{D}_{m}$.

Proposition 1. In a convex economy, competitive equilibria exist.

Proof: The set of prices of contracts is ${ }^{4} \mathcal{Q}=\Delta^{M-1}$.

The action correspondence of an individual, $\alpha^{i}$, is defined by

$$
\begin{gathered}
\alpha^{i}(q, R)= \\
\left\{(x, \theta, \phi, D \phi) \in \beta(q, R):\left(x^{\prime}, \theta^{\prime}, \phi^{\prime}, D^{\prime} \phi^{\prime}\right) \in \beta(q, R) \Rightarrow u^{i}\left(x^{\prime}\right) \leq u(x)\right\} ;
\end{gathered}
$$

the set $\alpha^{i}(q, R)$ is the set of actions that maximize the utility function of the individual over the budget set.

The compensated action correspondence of an individual, $\tilde{\alpha}^{i}$, is defined by

$$
\begin{gathered}
\tilde{\alpha}^{i}(q, R)=\{(x, \theta, \phi, D \phi) \in \beta(q, R): \\
\left.(\hat{x}, \hat{\theta}, \hat{\phi}, \hat{D} \hat{\phi}) \in \beta(q, R) \text {, and } q(\hat{\theta}-\hat{\phi})<0 \Rightarrow u^{i}(\hat{x}) \leq u(x)\right\} ;
\end{gathered}
$$

the complement of the set $\tilde{\alpha}^{i}(q, R)$ with respect to the budget set is the set,

$$
\begin{gathered}
\tilde{\alpha}_{c}^{i}(q, R)=\{(x, \theta, \phi, D \phi) \in \beta(q, R): \\
\left.u^{i}(\hat{x})>u(x) \text {, for some }(\hat{x}, \hat{\theta}, \hat{\phi}, \hat{D} \hat{\phi}) \in \beta(q, R), \text { such that } q(\hat{\theta}-\hat{\phi})<0\right\},
\end{gathered}
$$

of actions that are budget feasible but yield lower utility than some action that satisfies the budget constraint with strict inequality.

\footnotetext{
4 " $\Delta^{K}$ " denotes the simplex of dimension $K$.
} 
For $n=M+1, \ldots$, the truncated set of prices of contracts is $\mathcal{Q}_{n}=\{q \in$ $\left.\Delta^{M-1}: q_{m} \geq(1 / n), m \in \mathcal{M}\right\}$.

There exists a non-empty, compact, convex set $\mathcal{A}_{n}^{i}$, such that $\tilde{\alpha}^{i}(q, R) \in \mathcal{A}_{n}^{i}$, for $(q, R) \in \mathcal{Q}_{n} \times \mathcal{D}$; across individuals, $\mathcal{A}_{n}=\times_{i \in \mathcal{I}} \mathcal{A}_{n}^{i}$.

For $(q, R) \in \mathcal{Q}_{n} \times \mathcal{D}$, the budget set, $\beta^{i}(q, R)$, is non-empty and compact. Since the utility function is continuous, the set $\alpha^{i}(q, R)$ is non-empty and closed, and, hence, compact; since $\tilde{\alpha}^{i}(q, R)$ contains $\alpha^{i}(q, R)$, it is non-empty, and, since its complement, $\tilde{\alpha}_{c}^{i}(q, R)$ is an open set, it is closed and, hence, compact.

The set $\tilde{\alpha}^{i}(q, R)$ is convex: if $\hat{a}, \hat{\hat{a}} \in \tilde{\alpha}^{i}(q, R)$, and $0 \leq \lambda \leq 1$, then $a=$ $\lambda \hat{a}+(1-\lambda) \hat{\hat{a}} \in \tilde{\alpha}^{i}(q, R)$. This follows from the quasi-concavity of the utility function, as long as $a \in \beta^{i}(q, R)$; but this is the case: it suffices to set $\phi_{m}=$ $\lambda \hat{\phi}_{m}+(1-\lambda) \hat{\hat{\phi}}_{m}$ and $d_{m}=\lambda\left(\hat{\phi}_{m} / \phi_{m}\right) \hat{d}_{m}+(1-\lambda)\left(\hat{\hat{\phi}}_{m} / \phi_{m}\right) \hat{\hat{d}}_{m}$.

The compensated action correspondence, $\tilde{\alpha}^{i}$, defined by $\tilde{\alpha}^{i}(q, R)$, is upper hemi-continuous on $\mathcal{Q}_{n} \times \mathcal{D}$. If a sequence $\left((q, R)_{k} \in \mathcal{Q}_{n} \times \mathcal{R}: k=1, \ldots\right)$ converges: $\lim _{k \rightarrow \infty}(q, R)_{k}=(q, R)$, an associated sequence of actions $\left(a_{k}=\right.$ $\left.(x, \theta, \phi, D \phi)_{k}: k=1, \ldots\right)$ is bounded and, without loss of generality it converges: $\lim _{k \rightarrow \infty} a_{k}=a=(x, \theta, \phi, D \phi)$. If $a \notin \tilde{\alpha}^{i}(q, R)$, then there exists $\hat{a}=(\hat{x}, \hat{\theta}, \hat{\phi}, \hat{D} \hat{\phi}) \in \beta^{i}(q, R)$, such that $q(\hat{\theta}-\hat{\phi})<0$, and $u(\hat{x})>u(x)$. If the sequence $\left(a_{k}^{\prime}=\left(\hat{x}_{k}, \hat{\theta}, \hat{\phi}, \hat{D} \hat{\phi}\right): k=1, \ldots\right)$ is defined by $\hat{x}_{k}=e^{i}+R_{k} \hat{\theta}-\hat{D} \hat{\phi}$, by the continuity of the utility function, there exists $\bar{k}$, such that $u^{i}\left(\hat{x}_{k}\right)>u^{i}\left(x_{k}\right)$, for $k=\bar{k}, \ldots$ Since $a_{k} \in \tilde{\alpha}^{i}\left(q_{k}, R_{k}\right), q_{k}(\hat{\theta}-\hat{\phi}) \geq 0$, and, as a consequence, $q(\hat{\theta}-\hat{\phi}) \geq 0$, which contradicts $q(\hat{\theta}-\hat{\phi})<0$, for $k=\bar{k}, \ldots$

The correspondence $\psi_{n}=\left(\psi_{n}^{1}, \psi_{n}^{2}, \psi_{n}^{3}\right)$, with domain and range $\mathcal{Q}_{n} \times \mathcal{D} \times \mathcal{A}_{n}$, is defined component-wise, by

$$
\begin{aligned}
\psi_{n}^{1}(q, R, a)= & \arg \max \left\{q\left(\sum_{i \in \mathcal{I}} \theta^{i}-\sum_{i \in \mathcal{I}} \phi^{i}\right): q \in \mathcal{Q}_{n}\right\}, \\
\psi_{n, m}^{2}(q, R, a)= & \left(\sum_{i \in \mathcal{I}}\left((1 / n)+\phi_{m}^{i}\right)\right)^{-1} \sum_{i \in \mathcal{I}} d_{m}^{i}\left((1 / n)+\phi_{m}^{i}\right), \\
& m \in \mathcal{M}, \\
\psi_{n}^{3}(q, R, a)= & \times_{i \in I} \tilde{\alpha}^{i}(q, R) ;
\end{aligned}
$$

in particular, $\psi_{n, m^{*}}^{2}(q, R, a)=\mathbf{1}_{l^{*}}^{L}$, since $d_{m^{*}}^{i}=\mathbf{1}_{l^{*}}^{L}$.

The correspondence $\psi_{n}$ is non-empty, compact, convex, valued and upper hemi-continuous, and, therefore, admits a fixed point, $\left(q^{*}, R^{*}, a^{*}\right)_{n}$.

The sequence of fixed points $\left(\left(q^{*}, R^{*}, a^{*}\right)_{n}: n=M+1, \ldots\right)$, converges: $\lim _{n \rightarrow \infty}\left(q^{*}, R^{*}, a^{*}\right)_{n}=\left(q^{*}, R^{*}, a^{*}\right)$.

From the monotonicity of the utility function in the payoff of contract $m^{*}$, the value of the sales of contracts coincides with the value of the purchases of contracts for every individual, and summation across individuals yields that $q_{n}^{*}\left(\sum_{i \in \mathcal{I}} \theta^{i *}-\sum_{i \in \mathcal{I}} \phi^{i *}\right)_{n}=0$. From the definition of $\psi_{n}^{1}$, it follows that $q\left(\sum_{i \in \mathcal{I}} \theta^{i} *-\sum_{i \in \mathcal{I}} \phi^{i *}\right)_{n}=0$, for all $q \in \mathcal{Q}_{n}$, and, in particular, for $q=$ $(1 / M, \ldots, 1 / M)$, which implies that $\sum_{m \in \mathcal{M}} \sum_{i \in \mathcal{I}} \theta_{m, n}^{i *} \leq \sum_{m \in \mathcal{M}} \sum_{i \in \mathcal{I}} \phi_{m, n}^{i *}$; since the sales of contracts lie in a compact set, without loss of generality, for 
individual $i$, portfolios of contracts purchased converge to $\theta^{i *}$. This, in turn, implies that the consumption bundle converges, to $x^{i *}$. The sequence of actions $\left(a_{n}^{*}: n=M+1, \ldots\right)$ thus converges: $\lim _{n \rightarrow \infty} a_{n}^{*}=a^{*}$.

At the profile of actions $a^{*}$, markets for contracts clear. Taking limits, $q\left(\sum_{i \in \mathcal{I}} \theta^{i *}-\sum_{i \in \mathcal{I}} \phi^{i *}\right) \leq 0$, for all $q \in \cup_{n=M+1, \ldots} \mathcal{Q}_{n}$, which implies that $\left(\sum_{i \in \mathcal{I}} \theta^{i *}-\sum_{i \in \mathcal{I}} \phi^{i *}\right) \leq 0$. If, for some contract, $\left(\sum_{i \in \mathcal{I}} \theta_{m}^{i *}-\sum_{i \in \mathcal{I}} \phi_{m}^{i *}\right)<0$, a modification of the demand of some individual, to $\theta_{m}^{i *}-\left(\sum_{i \in \mathcal{I}} \theta_{m}^{i *}-\sum_{i \in \mathcal{I}} \phi_{m}^{i *}\right)$ assures market clearing.

The state of the economy $\left(R^{*}, a^{*}\right)$ is feasible. At a fixed point,

$r_{l, m, n}^{*}=\left(\sum_{i \in \mathcal{I}}\left((1 / n)+\phi_{m, n}^{i *}\right)\right)^{-1} \sum_{i \in \mathcal{I}} d_{l, m, n}^{i *}\left((1 / n)+\phi_{m, n}^{i *}\right), \quad l \in \mathcal{L}, m \in \mathcal{M} \backslash\left\{m^{*}\right\}$.

By direct substitution,

$$
\sum_{i \in \mathcal{I}} x_{n}^{i *}-\sum_{i \in \mathcal{I}} e^{i}=R_{n}^{*}\left(\sum_{i \in \mathcal{I}} \theta_{n}^{i *}-\sum_{i \in \mathcal{I}} \phi_{n}^{i *}\right)+\sum_{i \in \mathcal{I}}\left(D_{n}^{i *}-R_{n}^{*}\right) \mathbf{1}^{M}(1 / n),
$$

and the right hand side converges to zero.

For every individual, $a^{i *}=\left(x^{i *}, \theta^{i *}, \phi^{i *}, D^{i *} \phi^{i *}\right) \in \tilde{\alpha}^{i}\left(q^{*}, R^{*}\right)$. If $\hat{a}^{i} \in \beta^{i}\left(q^{*}\right.$, $\left.R^{*}\right)$ is such that $u^{i}\left(\hat{x}^{i}\right)>u^{i}\left(x^{i *}\right)$, while $q^{*}\left(\hat{\theta}^{i}-\hat{\phi}^{i}\right)<0$, then by the continuity of the utility function, there exists $\bar{n}$, such that $u^{i}\left(\hat{x}^{i}\right)>u^{i}\left(x_{n}^{i *}\right)$, for $n>\bar{n}$. The sequence $\left(\hat{a}_{n}^{i}: n=M+1, \ldots\right)$ defined by $\hat{x}_{n}^{i}=e^{i}+R_{n}^{*} \hat{\theta}^{i}-\hat{D}^{i} \hat{\phi}^{i}$ converges: $\lim _{n \rightarrow \infty} \hat{x}_{n}^{i}=\hat{x}^{i}$; by the continuity of the utility function, there exists $\overline{\bar{n}}$, such that $u^{i}\left(\hat{x}_{n}^{i}\right)>u^{i}\left(x_{n}^{i *}\right)$, for $n>\overline{\bar{n}}$. Since $\hat{a}_{n}^{i} \in \tilde{\alpha}^{i}\left(q_{n}^{*}, R_{n}^{*}\right), q_{n}^{*}\left(\hat{\theta}^{i}-\hat{\phi}^{i}\right) \geq 0$, which contradicts $q^{*}\left(\hat{\theta}^{i}-\hat{\phi}^{i}\right)<0$.

The price of contract $m^{*}$ is positive: $q_{m^{*}}^{*}>0$. If $q_{m^{*}}^{*}=0$, there exists a contract, $\hat{m} \in \mathcal{M} \backslash\left\{m^{*}\right\}$, with positive price: $q_{\hat{m}}^{*}>0$. Either, for every individual, $\phi_{\hat{m}}^{i *}=0$ or, for some individual, $\hat{i}, \phi_{\hat{m}}^{\hat{i} *}>0$. Since the utility function of every individual is strictly monotonically increasing in the payoff of contract $m^{*}, u^{i}\left(x^{i *}+\mathbf{1}_{l^{*}}^{L}\right)>u^{i}\left(x^{i *}\right)$. Since $\phi_{\hat{m}}^{i *}>0$, there exists $\bar{n}$, such that, for $n \geq \bar{n}$, $u^{i}\left(x^{i *}+\mathbf{1}_{l^{*}}^{L}-d_{\hat{m}}^{i *}(1 / n)\right)>u^{i}\left(x^{i *}\right) ;$ but $q^{*}\left(\mathbf{1}_{m^{*}}^{M}-\mathbf{1}_{\hat{m}}^{M}(1 / n)\right)<0$, which contradicts $a^{\hat{i} *} \in \tilde{\alpha}^{\hat{i}}\left(q^{*}, R^{*}\right)$. If, for every individual, $\phi_{\hat{m}}^{i *}=0$, a contradiction follows, since there exists an individual who can sell the contract $\hat{m}: e^{i} \geq d_{\hat{m}} \phi_{\hat{m}}$, for some $\left(d_{\hat{m}}, \phi_{\hat{m}}\right) \in \mathcal{D}_{\hat{m}} \times \Phi_{\hat{m}}$, with $\phi_{\hat{m}}>0$.

For every individual, $a^{i *}=\left(x^{i *}, \theta^{i *}, \phi^{i *}, D^{i *} \phi^{i *}\right) \in \alpha^{i}\left(q^{*}, R^{*}\right)$. The action $\hat{a}^{i}=\left(\hat{x}^{i}, \hat{\theta}^{i}, \hat{\phi}^{i}, D^{i *} \hat{\phi}^{i}\right)$, with $\hat{x}^{i}=e^{i}+R^{*} \hat{\theta}^{i}-D^{i *} \hat{\phi}^{i}, \hat{\theta}^{i}=0$, and $\hat{\phi}^{i}=\mathbf{1}_{m^{*}}^{M} e_{l^{*}}^{i}$, satisfies $\hat{a}^{i} \in \beta^{i}\left(q^{*}, R^{*}\right)$ and $q^{*}\left(\hat{\theta}^{i}-\hat{\phi}^{i}\right)=-q_{m^{*}}^{*} e_{l^{*}}^{i}<0$. If $a^{i \prime} \in \beta^{i}\left(q^{*}, R^{*}\right)$ is such that $u^{i}\left(x^{i \prime}\right)>u^{i}\left(x^{i *}\right)$, then setting $a_{\lambda}^{i}=(1-\lambda) a^{i \prime}+\lambda \hat{a}^{i}$, for $0<\lambda<1$, by the continuity of the utility function, contradicts $a^{i *} \in \tilde{\alpha}^{i}\left(q^{*}, R^{*}\right)$, for $\lambda$ sufficiently small.

The scope of the analysis is enlarged by allowing individuals access to private technologies, $\mathcal{Y}^{i} \subset \mathcal{R}^{L} \times \mathcal{R}^{L}$, that can be employed to transform endowments prior to exchange. 
Assumption 5. For every individual, the technology is compact and it does not allow for the transformation of commodity $l^{*}:$ if $\left(e^{i}, y^{i}\right) \in \mathcal{Y}^{i}, y_{l^{*}}^{i}=e_{l^{*}}^{i}$.

In a convex economy, for every individual, the technology $\mathcal{Y}^{i}$ is convex.

An action by an individual, at payoffs of contracts $R$, is $a^{i}=\left(x^{i}, y^{i}, \theta^{i}, \phi^{i}\right.$, $\left.D^{i} \phi^{i}\right)$, such that

- $x^{i}=y^{i}+\left(R \theta^{i}-D^{i} \phi^{i}\right) \in \mathcal{X}^{i}$,

- $\left(e^{i}, y^{i}\right) \in \mathcal{Y}^{i}$.

The definition of a competitive equilibrium for the model with production is a standard extension of the previous one. The existence of competitive equilibria for a convex economy follows by an immediate generalization of the earlier argument.

\subsection{Examples}

The model encompasses instances of economies with asymmetric information, as well as economies where the discretion over deliveries is generated by limited commitment, as in models of default, price rigidities or incompleteness of contracts .

In simple economies, one commodity, "money" or consumption at the contracting stage trades directly; it corresponds to commodity $l^{*}$ in the formal model and is often not mentioned explicitly. Also, when all other commodities, usually $l=1,2$, trade through a single contract, the index " $m$ " that identifies contracts is omitted.

Adverse selection occurs when the privately observed, but fixed characteristics of the sellers, preferences or endowments, determine their deliveries on contracts.

In the market for "lemons" in Akerlof (1974), each seller is endowed with and can deliver either a car of high quality, commodity 1 , or a car of low quality, commodity 2 .

A contract for the sale of a car is described by the delivery set

$$
\mathcal{D}=\left\{d \geq 0: d_{1}+d_{2}=1\right\} .
$$

The constraints

$$
\begin{gathered}
e_{1}^{i}-d_{1}^{i} \phi^{i} \geq 0, \\
e_{2}^{i}-d_{2}^{i} \phi^{i} \geq 0
\end{gathered}
$$

imply that the informational advantage of sellers pertains exclusively to the cars in their endowment: individuals are not distinguished by their ability to recognize the quality of the engines of cars traded in the market. Sellers with endowments $e_{2}^{i}=0$ are only able to sell cars of high quality. 
Similarly, in the insurance market in Rothschild and Stiglitz (1976), commodities 1 and 2 represent "future consumption at state 1" and "future consumption at state 2 ," respectively. State 1 occurs with high probability, $\pi_{1}>$ $(1 / 2)$.

Damage payments on insurance contract are restricted to the set

$$
\mathcal{D}=\left\{d \geq 0: d_{1}+d_{2}=1\right\} .
$$

Individuals who suffer a loss at state 2, make premium payments at state 1 , which occurs with higher probability; they represent "good risk."

Moral hazard occurs when unobservable choices by the seller, rather than his characteristics, determine deliveries on contracts.

In the insurance market in Grossman and Hart (1983) deliveries on contracts are restricted to the set

$$
\mathcal{D}=\left\{d \geq 0: d_{1}+d_{2}=1\right\},
$$

where, again, commodities 1 and 2 represent "future consumption at state 1 " and "future consumption at state 2 ," respectively, and state 1 occurs with high probability, $\pi_{1}>(1 / 2)$.

Here, however, individuals are endowed with a technology,

$$
\mathcal{Y}=\left\{\left(e_{3}, y_{1}\right) \geq 0: y_{1}=k e_{3}\right\},
$$

that transforms a third commodity, "leisure," into units of consumption at the high probability state.

The conditions

$$
\begin{gathered}
y_{1}^{i}+e_{1}^{i}-d_{1}^{i} \phi^{i} \geq 0, \\
e_{2}^{i}-d_{2}^{i} \phi^{i} \geq 0
\end{gathered}
$$

guarantee that deliveries must come out of the endowments of individuals, as transformed by the production activity, and hence depend on production choices of sellers.

Exclusive contractual relationships in an insurance market with moral hazard or adverse selection allow contracts to be differentiated according to the quantity traded. There is a large number, $k \in \mathcal{K}=\{1, \ldots, K\}$, of contracts; all contracts have the same set of deliveries

$$
\mathcal{D}=\left\{d \geq 0: d_{1}+d_{2}=1\right\} ;
$$

they are distinguished by their sets of admissible sales

$$
\Phi^{k}=\{0, k\} .
$$


Across contracts, the set of admissible sales of portfolios is

$$
\Phi=\left\{\phi \in \times_{k \in \mathcal{K}} \Phi^{k}: \phi^{k}=k \Rightarrow \phi^{j}=0, \text { for } j \neq k\right\} .
$$

The specification, which violates the product structure of the general specification, implies that individuals can only trade a non-zero amount of only one contract.

In this set-up, the pricing of contracts is effectively non-linear.

Though the set of equilibria can be large, refinements, as in Dubey, Geanakoplos, Shubik (1990, 2005) or Gale (1992)) yield the same set of equilibria as in Prescott and Townsend (1984).

Default is possible when sellers of a contract have the option of exchanging utility penalties for payments of debts, as in Dubey, Geanakoplos and Shubik $(1990,2005)$. If payments are denominated in commodity 1 , while penalties are denominated in commodity 2 , the set of deliveries is

$$
\mathcal{D}=\left\{d \geq 0: d_{1}+\lambda d_{2}=1\right\},
$$

where $\lambda>0$ measures the severity of the penalty; utility penalties are possibly paid by debtors in a commodity whose consumption is of no interest to creditors.

In the case of collateral, as in Dubey, Geanakoplos and Zame (1996), creditors derive utility from the consumption of the penalty commodity.

Signaling occurs in a market with adverse selection, when sellers of a commodity of high quality or good risks are the only individuals endowed with a third commodity, the "ability to acquire education," as in Spence (1974). A contract with signaling requires the delivery of the signaling commodity, 3 , and

$$
\mathcal{D}=\left\{d>0: d_{1}+d_{2}=1, d_{3}=1\right\} .
$$

Ownership and control, following Grossman and Hart (1986) may influence the nature of contracts. Commodity 1 is the consumption good and commodity 2 is the collateral good. There are two possible states of uncertainty. Contract $(m, s)$ accounts for the direct trade of commodity $m$ in state of the world $s$. The relative price of the collateral good in state $s$ is $\frac{q_{2 s} s}{q_{1 s}}$. Control rights are allocated to the seller if , as before, the set of deliveries of a collateralized debt contract is

$$
\mathcal{D}=\left\{\left(d_{1 s}, d_{2 s}\right)_{s=\alpha, \beta} \geq 0 ; d_{1 s}+\lambda d_{2 s}=1\right\} .
$$

When control rights are allocated to the buyer,

$$
\mathcal{D}=\left\{\left(d_{1 s}, d_{2 s}\right)_{s=\alpha, \beta} \geq 0: d_{1 s}+\lambda d_{2 s}=1, d_{2 s}>0 \text { if } \frac{q_{2 s}}{q_{1 s}}>\lambda\right\} .
$$


The buyer accepts the delivery of the collateral good only in the state of the world in which such good is worth more than the exchange rate implicit in the contract, $\lambda$.

The possibility of renegotiation, as in Hart and Moore (1988), can be similarly dealt with in this set-up.

Price rigidities and rationing occur when the price of a commodity, 1, is required to equal the price of another commodity, 2. Buyers receive a given composition of commodities 1 and 2 and are typically rationed in the commodity of most desired quality.

\section{$3 \quad$ Efficiency}

The determination of the payoffs of contracts by the choices of sellers at equilibrium creates an externality, which is a source of inefficiency.

A state of the economy, $(R, a)$, is incentive-compatible if, for every individual, $u^{i}\left(e^{i}+R \theta^{i}-D^{i} \phi^{i}\right) \geq u^{i}\left(e^{i}+R \theta^{i}-D \phi^{i}\right)$, for all $D$ such that $\left(e^{i}+R \theta^{i}-D \phi^{i}\right) \in \mathcal{X}^{i}$.

A feasible and incentive-compatible state of the economy is incentive-efficient if no feasible and incentive-compatible state is Pareto superior.

Prescott and Townsend (1984) showed that incentive-efficient allocations obtain as equilibria with appropriate restrictions over trades.

Incentive-efficiency restricts attention to interventions compatible with the discretion of sellers over deliveries on contracts; but it requires full controllability of individual trades, which is not satisfactory when trade takes place on large, anonymous markets.

Greenwald and Stiglitz (1986) proposed the taxation of contracts and anonymous, lump-sum transfers as the appropriate intervention in a market economy under asymmetric information and incentive compatibility constraints; it does not require the trades or characteristics of individuals to be observable.

A fiscal authority imposes ad-valorem taxes on the sales of contracts, $t=$ $\left(\ldots, t_{m}, \ldots\right)$, and redistributes revenue, $T$, to each individual.

At prices of assets and taxes $(q, t, T)$, the budget constraint of an individual is

$$
q \theta-(q+t) \phi-T \leq 0
$$

the budget constraint of the fiscal authority is

$$
t \sum_{i \in \mathcal{I}} \phi^{i}+I T \leq 0 .
$$

A competitive equilibrium with taxation is $\left(q^{*}, t^{*}, T^{*}, R^{*}, a^{*}\right)$.

Taxation implements a state of the economy, $(\hat{R}, \hat{a})$, if there exist prices of assets and taxes, $(\hat{q}, \hat{t}, \hat{T})$, such that $(\hat{q}, \hat{t}, \hat{T}, \hat{R}, \hat{a})$ is a competitive equilibrium with taxation. 
Definition 2. A competitive equilibrium is constrained suboptimal if there is a Pareto superior state of the economy $(R, a)$, implementable by taxes $(t, T)$.

In what follows we focus attention on economies with adverse selection, in which the deliveries on contracts, $D^{i}$, are an exogenous characteristic of each individual. This makes the argument establishing generic constrained inefficiency both simpler and clearer. Such argument follows the one used by Geanakoplos and Polemarchakis (1986) and Citanna, Kajii and Villanacci (1998) for economies with an incomplete asset market.

In recent work, Geanakoplos and Polemarchakis (2008) showed that the taxation of exchanges implements Pareto improvements in abstract economies with externalities, while Citanna, Polemarchakis and Tirelli (2006) obtained an analogous result for economies with an incomplete asset market.

In a smooth, convex economy, for every individual, the utility function, $u^{i}$, is twice continuously differentiable on the interior of the consumption set; $D u^{i}$ is strictly positive, while $D^{2} u^{i}$ is negative definite on the orthogonal complement of $D u^{i}$; the endowment, $e^{i}$, is strictly positive and strictly preferred to any consumption plan on the boundary of the consumption set.

With taxation, the first order conditions for an interior optimum of the individual optimization problem are

$$
\begin{aligned}
& D u^{i}-\mu^{i}=0, \\
& R^{\prime} \mu^{i \prime}-q^{\prime} \lambda^{i}=0, \\
& -D^{i \prime} \mu^{i \prime}+\left(q^{\prime}+t^{\prime}\right) \lambda^{i}=0, \\
& -x^{i}+e^{i}+R \theta^{i}-D^{i} \phi^{i}=0, \\
& -q \theta^{i}+(q+t) \phi^{i}+T=0,
\end{aligned}
$$

where $\mu^{i}=\left(\ldots, \mu_{l}^{i}, \ldots\right)$ are the strictly positive Lagrange multipliers associated with the constraints $-x+e^{i}+R \theta^{i}-D^{i} \phi^{i}=0$, and $\lambda^{i}$ is the positive Lagrange multiplier associated with the constraint $q \theta^{i}-(q+t) \phi^{i}-T=0$.

The market clearing conditions are

$$
\begin{aligned}
& \sum_{i \in \mathcal{I}}\left(\hat{\theta}^{i}-\hat{\phi}^{i}\right)=0, \\
& \sum_{i \in \mathcal{I}} \tilde{\Phi}^{i} \tilde{R}-\sum_{i \in \mathcal{I}} \tilde{\Phi}^{i} \tilde{D}^{i}=0, \\
& t \sum_{i \in \mathcal{I}} \phi^{i}+I T=0,
\end{aligned}
$$

where $\hat{\theta}^{i}=\left(\theta_{1}^{i}, \ldots, \theta_{M-1}^{i}\right), \hat{\phi}^{i}=\left(\phi_{1}^{i}, . ., \phi_{M-1}^{i}\right) ; \tilde{\Phi}^{i}$ is an $(L M \times L M)$ diagonal matrix with elements $\tilde{\Phi}_{l m, l m}^{i}=\phi_{m}^{i} ; \tilde{R}$ and $\tilde{D}^{i}$ are $L M$-vectors $\left(\ldots, r_{l m}, \ldots\right)$, $\left(\ldots, d_{l m}, \ldots\right)$.

Differentiating the above equations one obtains, by repeated substitution, that 


$$
\begin{gathered}
\sum_{i} \frac{D u^{i}}{\lambda^{i}} d x^{i}=\sum_{m} \sum_{i} \theta_{m}^{i} \frac{D u^{i}}{\lambda^{i}} d r_{m}= \\
=-\sum_{m} q_{m} \sum_{i} d \theta_{m}^{i}+\sum_{m} \frac{1}{\sum_{i} \theta_{m}^{i}} \sum_{i} \theta_{m}^{i}\left(\sum_{j} \frac{D u^{i}}{\lambda^{i}} d_{m}^{j} d \phi_{m}^{j}\right) .
\end{gathered}
$$

A necessary condition for a Pareto improvement to exist is that this sum be different from zero. It is immediate that this necessary condition is violated if, at equilibrium, the normalized gradients of the utility functions of individuals are collinear or if the delivery choices of individuals are similar, so that

$$
\frac{D u^{i}}{\lambda^{i}} d_{m}^{j}=q_{m}, \quad \text { for all } i, j \text { and } m \text {. }
$$

Thus, for Pareto improvement it is necessary that individuals be sufficiently diverse in their deliveries, in a sense to be made precise, while the market is sufficiently incomplete.

The way to an improvement is via changes in the matrix of payoffs, $R$, induced by changes in the supplies of sellers, $\phi^{i}$ - the externality discussed earlier. Even if the deliveries of individuals are different, aggregate payoffs can be modified in sufficiently many directions by taxes and transfers only if the reactions of sellers to changes in taxation do not offset each other. For this, it is necessary to perturb the second derivatives of utility functions around the equilibrium.

Assumption 6. For every individual, everywhere in the interior of the consumption set and for every $b_{x} \neq 0$, the subspace ${ }^{5}$

$$
\left[D_{\gamma^{i}}\left(D_{x, x}^{2} u^{i} b_{x}\right) y: D_{\gamma^{i}, x}^{2} u^{i} y=0\right],
$$

has full dimension, $L$.

This ensures that, at an interior allocation, it is possible to perturb fully the matrix of second derivatives of the utility functions of individuals, while leaving the first derivatives unaffected, as perturbations along the set of competitive equilibrium prices and allocations require; Citanna, Kajii and Villanacci (1998) developed the construction in full.

An economy is described by $\omega=\left(\ldots,\left(e^{i}, \gamma^{i}\right), \ldots\right)$, where $\gamma^{i}$, a vector of dimension, $\Gamma^{i}$ parameterizes the utility function of agent $i ; \gamma=\left(\ldots, \gamma^{i}, \ldots\right)$, and $\Gamma=\sum_{i \in \mathcal{I}} \Gamma^{i}$. The set of economies is an open set, $\Omega$, of dimension $I L+\Gamma$.

\footnotetext{
5 "[ ]" denotes the subspace spanned by a collection of vectors or the column span of a matrix.
} 
The function $G$ is defined by

$$
G(\xi, \tau, \omega)=\left(\begin{array}{c}
\vdots \\
F^{i} \\
\vdots \\
E
\end{array}\right),
$$

where $\xi=\left(\ldots,\left(x^{i}, \theta^{i}, \phi^{i}, \mu^{i}, \lambda^{i}\right), \ldots, \hat{q}, R\right)$, a vector of dimension $n=I(2 L+$ $2 M+1)+(M-1)+L M$, and $\tau=(\hat{t}, T)$, a vector of dimension $M$. The equations $F^{i}=0$ are the first order conditions for a solution to the individual optimization problem, while $E=0$ are the market clearing conditions at a competitive equilibrium with taxation. The analogous function without taxation, where $\tau=0$, and the last equation in $E$ is dropped, is $\hat{G}(\xi, \omega)$.

Interior competitive equilibria, $\xi$, of an economy, $\omega$, are solutions of $\hat{G}(\xi, \omega)=$ 0 , with all variables in the interior of their domain of definition.

A regular interior competitive equilibrium is such that $\operatorname{dim}\left[D_{\xi} \hat{G}\right]=n$.

Definition 3. A competitive equilibrium of a smooth, convex economy displays sufficient diversity of individuals if

1. for every individual, $\operatorname{dim}\left[R, D^{i}\right]=2 M$;

2. for every contract, $\operatorname{dim}\left[\beta_{i, j}=\theta_{m}^{j} \frac{D u^{j}}{\lambda^{j}}\left[r_{m}-d_{m}^{i}\right]:(i, j) \in \mathcal{I} \times \mathcal{I}\right]=I$.

The first condition implies that, at the competitive equilibrium, deliveries made by individuals are sufficiently different so that they are never collinear to average deliveries, while the second requires that there be sufficient diversity among individuals. In particular, if gradients were collinear, $\left(D u^{j} / \lambda^{j}\right)=$ $\left(D u^{i} / \lambda^{i}\right)$ or if deliveries were not differentiated, $d^{j}=d^{i}$, the elements $\beta_{i, j}$ would be null.

Proposition 2. In a smooth, convex economy with adverse selection, if

1. $M>I, L>2 M$, and

2. for an open set of economies, competitive equilibria are regular interior and display sufficient diversity of individuals ${ }^{6}$,

then, for an open and dense subset of this set of economies, competitive equilibria are constrained suboptimal.

\footnotetext{
${ }^{6}$ In condition 2. we assume the existence of an open set of economies whose equilibria are regular and interior. This is a stronger assumption than needed, it would be enough to assume the existence of an open set of economies whose equilibria are regular, without requiring interiority. The existence of such an open set of economies has been proved in similar settings (e.g. with short sales constraints and endogenous asset payoffs) by Geanakoplos, Magill, Quinzii and Drèze (1990); see also Villanacci et al. (2002), chapter 14.
} 
Proof: The function $H$ is defined by

$$
H(\xi, b, \omega)=\left(\begin{array}{c}
\hat{G}(\xi, \omega) \\
\left.D_{\xi, \tau}(G(\xi, 0, \omega), U(\xi, 0, \omega))^{\prime} b\right) \\
\|b\|-1
\end{array}\right),
$$

where $b=\left(\ldots,\left(b_{x}^{i}, b_{\theta}^{i}, b_{\phi}^{i}, b_{\mu}^{i}, b_{\lambda}^{i}\right), \ldots, b_{q}, b_{R}, b_{T}, \ldots, b_{u}^{i}, \ldots\right)$ is of dimension $n+$ $1+I$, while $U\left(\xi, 0, \omega=\left(\ldots, u^{i}\left(x^{i}\right), \ldots\right)\right.$. If the function $H$ is transverse to 0 , the result follows. This is the case since, for a given $\omega$, the number of equations, $(n+(n+M)+1)$, is greater than the number of unknowns, $(n+(n+1+I))$. As a consequence, if $H$ is transverse to 0 , for a generic set of $\omega$ there is no $(\xi, b)$ at which $H(\xi, b, \omega)=0$; that is, at an equilibrium $\xi$, the $((n+1+I) \times(n+$ $M)$ ) matrix $D_{\xi, \tau}(G, U)$ has full row rank. In particular, a Pareto improving intervention exists; again, Citanna, Kajii and Villanacci (1998) developed the construction in full.

The function $H$ is transverse to 0 if the Jacobian matrix

$$
D H=\left(\begin{array}{ccc}
D_{\xi} \hat{G} & 0 & D_{\omega} \hat{G} \\
D_{\xi}\left(D_{\xi, \tau}(G, U)^{\prime} b\right) & D_{\xi, \tau}(G, U)^{\prime} & D_{\omega}\left(D_{\xi, \tau}(G, U)^{\prime} b\right) \\
0 & b^{\prime} & 0
\end{array}\right)
$$

has full row rank whenever $H(\xi, b, \omega)=0$.

The columns of $D_{\omega} \hat{G}$ that correspond to derivatives with respect to $\gamma$ are $D_{\gamma} \hat{G}$; the only non-zero elements are $D_{\gamma^{i}, x}^{2} u^{i}$. Similarly, in $D_{\gamma}\left(D_{\xi, \tau}(G, U)^{\prime} b\right)$, the only non-zero elements are $D_{\gamma^{i}}\left(D_{x, x}^{2} u^{i} b_{x}^{i}\right)$ and $D_{\gamma^{i}, x}^{2} u^{i} b_{u}^{i}$. Under assumption 8 , one can restrict attention to perturbations such that, for every individual, $D_{\gamma^{i}, x}^{2} u^{i}=0$.

At a regular equilibrium, $D_{\xi} \hat{G}$ has rank $n$. The problem then reduces to showing that, whenever $H(\xi, b, \omega)=0$, the matrix

$$
K=\left(\begin{array}{cc}
D_{\xi, \tau}(G, U)^{\prime} & D_{\gamma}\left(D_{\xi, \tau}(G, U)^{\prime} b\right) \\
b^{\prime} & 0
\end{array}\right)
$$

has full row rank, where the columns of $D_{\gamma}\left(D_{\xi, \tau}(G, U)^{\prime} b\right)$ corresponding to $\gamma^{i}$ are of the form $D_{\gamma^{i}}\left(D_{\xi, \tau}(G, U)^{\prime} b\right)=\left(0, \ldots, 0, D_{\gamma^{i}}\left(D_{x, x}^{2} u^{i} b_{x}^{i}\right), 0, \ldots, 0\right)^{\prime}=$ $\left(0, \ldots, 0, N^{i}(b), 0, \ldots, 0\right)^{\prime}$.

For full row rank of $K$, it suffices that any $z=K \Delta$ can be generated by an appropriate choice of

$$
\Delta=\left(\ldots,\left(\Delta_{x}^{i}, \Delta_{\theta}^{i}, \Delta_{\phi}^{i}, \Delta_{\mu}^{i}, \Delta_{\lambda}^{i}\right), \ldots, \Delta_{q}, \Delta_{R}, \Delta_{T}, \ldots, \Delta_{u}^{i}, \ldots, \ldots, \Delta_{\gamma}^{i}, \ldots\right) ;
$$


explicitly,

$$
\begin{aligned}
& z_{1 . i}=D^{2} u^{i} \Delta_{x}^{i}-I^{M} \Delta_{\mu}^{i}+D u^{i} \Delta_{u}^{i}+N^{i}(b) \Delta_{\gamma}^{i}, \\
& z_{2 . i}=R^{\prime} \Delta_{\mu}^{i}-q^{\prime} \Delta_{\lambda}^{i}+\hat{I}^{M} \Delta_{q}, \\
& z_{3 . i}=-D^{i \prime} \Delta_{\mu}^{i}+q^{\prime} \Delta_{\lambda}^{i}-\hat{I}^{M} \Delta_{q}+\left(\bar{R}^{\prime}-\bar{D}^{i^{\prime}}\right) \Delta_{R}, \\
& z_{4 . i}=-I^{M} \Delta_{x}^{i}+R \Delta_{\theta}^{i}-D^{i} \Delta_{\phi}^{i}, \\
& z_{5 . i}=-q \Delta_{\theta}^{i}+q \Delta_{\phi}^{i}, \\
& z_{6}=-\sum_{i \in \mathcal{I}}\left(\Lambda^{i} \Delta_{\theta}^{i}\right)+\sum_{i \in \mathcal{I}}\left(\Lambda^{i} \Delta_{\phi}^{i}\right)-\sum_{i \in \mathcal{I}}\left(\hat{\theta}^{i}-\hat{\phi}^{i}\right) \Delta_{\lambda}^{i}, \\
& z_{7}=\sum_{i \in \mathcal{I}} M^{i \prime} \Delta_{\theta}^{i}+\sum_{i \in \mathcal{I}} \Theta^{i \prime} \Delta_{\mu}^{i}+\sum_{i \in \mathcal{I}} \tilde{\Theta}^{i \prime} \Delta_{R}, \\
& z_{8}=\sum_{i \in \mathcal{I}} \Lambda^{i} \Delta_{\phi}^{i}+\sum_{i \in \mathcal{I}} \phi^{i} \Delta_{\lambda}^{i}+\sum_{i \in \mathcal{I}} \phi^{i} \Delta_{\lambda}^{i}, \\
& z_{9}=\sum_{i \in \mathcal{I}} \Delta_{\lambda}^{i}+I \Delta_{T}, \\
& z_{10}=\sum_{i \in \mathcal{I}} b^{i} \Delta^{i}+b_{q} \Delta_{q}+b_{R} \Delta_{R}+b_{T} \Delta_{T}+\sum_{i \in \mathcal{I}} b_{u}^{i} \Delta_{u}^{i},
\end{aligned}
$$

where $\hat{I}^{M}$ is the $(M \times(M-1))$ matrix obtained by adding a last row of zeros to the $(M-1)$-dimensional identity; $\Lambda^{i}$ is the transpose of $\hat{I}^{M}$ multiplied by the scalar $\lambda^{i} ; M^{i}$ is an $(M \times L M)$ matrix whose $m$-th row is of the form $\left(0, \ldots, \mu^{i \prime}, \ldots, 0\right)$, with non-zero elements corresponding to the $m$-th block of columns; $\Theta^{i}$ is an $(L \times L M)$ matrix whose $m$-th block of columns is the $L$ dimensional identity times the scalar $\theta_{m}^{i} ; \bar{R}$ is an $(L M \times M)$, block diagonal matrix whose columns are of the form $\left(0, r_{1 m}, r_{2 m}, \ldots, r_{L m}, 0\right)$, and similarly for $\hat{D}^{i}$.

If $b_{x}^{i} \neq 0$, one can restrict attention to perturbations such that $N^{i}(b)=$ $D_{\gamma^{i}} D_{x, x}^{2} u^{i} b_{x}^{i}$ has full rank, $L$, so that the elements $z_{1 . i}$ can be controlled by $\Delta_{\gamma}^{i}$. If, on the other hand $b_{x}^{i}=0$, the matrix $N^{i}(b)$ vanishes, and perturbation of $\gamma^{i}$ have no effect.

First, $b_{x}^{i} \neq 0$, for all $i$.

The first seven elements of $z$ can be controlled using $\left(\left(\Delta^{i}\right)_{i \in I}, \Delta_{q}, \Delta_{R}\right)$, because the corresponding matrix of coefficients is the Jacobian $D_{\xi} \hat{G}$ which has full rank $n$ at a regular equilibrium. The problem then reduces to showing that the remaining three elements can be controlled independently.

To control $z_{8}$, one uses the first $(M-1)$ elements of $\Delta_{\phi}^{1}$. This upsets $z_{6}$ and the elements $z_{4.1}$ and $z_{5.1}$, corresponding to individual 1 . One uses $\Delta_{\phi_{M}}^{1}$ to readjust $z_{5.1}$, and $\Delta_{x}^{1}$ to readjust $z_{4.1}$. This last move upsets $z_{1.1}$, but $N^{1}(b)$ has full rank $L$ and one can adjust $\Delta_{\gamma}^{1}$. To readjust $z_{6}$ one uses the first $(M-1)$ elements of $\Delta_{\theta}^{1}$. This again moves $z_{4.1}$ and $z_{5.1}$, which can be undone as before. 
But it also moves $z_{7}$. To undo this, one needs to use $\Delta R$, which upsets $z_{3 . i}$ for all $i$. Here there is a problem: to adjust these elements one should use $\Delta_{\mu}^{i}$ which moves $z_{2 . i}$ and $z_{7}$ itself (and $z_{1 . i}$, but this can be undone by $\Delta_{\gamma}^{i}$ ). One needs an argument to show that one can move $\Delta_{\mu}^{i}$ and $\Delta_{R}$ jointly to control $z_{2 . i}, z_{3 . i}$ and $z_{7}$. At a regular equilibrium, this is true. One considers the $(2 M I+L M)$-dimensional subsystem of equilibrium equations

$$
\begin{gathered}
-x^{i}+e^{i}+R \theta^{i}-D^{i} \phi^{i}=0, \\
\sum_{i \in \mathcal{I}} \tilde{\Phi}^{i} \tilde{R}-\sum_{i \in \mathcal{I}} \tilde{\Phi}^{i} \tilde{D}^{i}=0,
\end{gathered}
$$

where, of the first equations, one keeps only those corresponding to $2 M$ linearly independent rows of $\left[R, D^{i}\right]$. Taking derivatives with respect to $\left(\left(\theta^{i}, \phi^{i}, e^{i}\right)_{i \in I}, R\right)$ one obtains a matrix of full row rank. But then, for generic endowments, the $(2 M I+L M)$ square matrix of derivatives with respect to $\left(\left(\theta^{i}, \phi^{i}\right)_{i \in \mathcal{I}}, R\right)$ is full rank. This is exactly the matrix that allows one to jointly control $z_{2 . i}, z_{3 . i}$ and $z_{7}$.

To finish the argument, $z_{9}$ can be controlled by $\Delta_{T}$ (this upsets $z_{8}$, which can be readjusted as before), and $z_{10}$ by $\Delta_{u}^{i}$ corresponding to $b_{u}^{i} \neq 0$ (this upsets $z_{1 . i}$, to be readjusted by $\Delta_{\gamma}^{i}$ ). That a $b_{u}^{i} \neq 0$ exists follows from the fact that, if for all $i b_{u}^{i}=0$, then $D_{\xi, \tau}(G(\xi, 0, \omega), U(\xi, 0, \omega))^{\prime} b=0$ and regularity of equilibrium imply $b=0$, which is impossible at a zero of $H$.

Second, for some, $i b_{x}^{i}=0$.

For any one of the (finitely many) possible cases, a perturbation argument similar to the one above is possible.

At a zero of $H$, it cannot be the case that for all $i b_{x}^{i}=0$. The equations in 
$H=0$ corresponding to $\left.D_{\xi, \tau}(G(\xi, 0, \omega), U(\xi, 0, \omega))^{\prime} b\right)=0$ are

$$
\begin{aligned}
& D^{2} u^{i} b_{x}^{i}-I^{M} b_{\mu}^{i}+D u^{i} b_{u}^{i} \quad=0, \\
& R^{\prime} b_{\mu}^{i}-q^{\prime} b_{\lambda}^{i}+\hat{I}^{M} b_{q} \quad=0, \\
& -D^{i \prime} b_{\mu}^{i}+q^{\prime} b_{\lambda}^{i}-\hat{I}^{M} b_{q}+\left(\bar{R}^{\prime}-\bar{D}^{i \prime}\right) b_{R}=0, \\
& -I^{M} b_{x}^{i}+R b_{\theta}^{i}-D^{i} b_{\phi}^{i}=0, \\
& -q b_{\theta}^{i}+q b_{\phi}^{i} \quad=0, \\
& \sum_{i \in \mathcal{I}} \Lambda^{i}\left(b_{\phi}^{i}-b_{\theta}^{i}\right)-\sum_{i \in \mathcal{I}}\left(\hat{\theta}^{i}-\hat{\phi}^{i}\right) b_{\lambda}^{i}=0, \\
& \sum_{i \in \mathcal{I}} M^{i \prime} b_{\theta}^{i}+\sum_{i \in \mathcal{I}} \Theta^{i \prime} b_{\mu}^{i}+\sum_{i \in \mathcal{I}} \tilde{\Theta}^{i} b_{R}=0, \\
& \sum_{i \in \mathcal{I}} \Lambda^{i} b_{\phi}^{i}+\sum_{i \in \mathcal{I}} \hat{\phi}^{i}\left(b_{\lambda}^{i}+b_{T}\right)=0, \\
& \sum_{i \in \mathcal{I}}\left(b_{\lambda}^{i}+b_{T}\right) \quad=0 .
\end{aligned}
$$

If $b_{x}^{i}=0$, the fourth equation and $\operatorname{dim}\left[R, D^{i}\right]=2 M$ immediately imply $b_{\theta}^{i}=b_{\phi}^{i}=0$. From the second, third and seventh equations, and the first order conditions,one obtains that, for all $i$ and all $m$,

$$
\sum_{j \in \mathcal{I}} \lambda^{j} b_{u}^{j} \frac{\theta_{m}^{j}}{\theta_{m}}\left[q_{m}-\frac{D u^{j}}{\lambda^{j}} d_{m}^{i}\right]=0
$$

this implies that $b_{u}^{i}=0$, for all $i$. But then, substitution in the above equations yields $b=0$, which is impossible at a zero of $H$.

If $b_{x}^{i}=0$ for $i \in \mathcal{I}_{k}$, a subset of individuals, then $b_{\theta}^{i}=b_{\phi}^{i}=0$, for all $i \in \mathcal{I}_{k}$. Moreover, from the second equation above and the first order conditions, $\lambda^{i} b_{u}^{i}-b_{\lambda}^{i}=0$. One can then write a new system of equations $H_{k}=0$, in which, for each $i \in \mathcal{I}_{k}$, one adds these $L+2 M+1$ equations and drops those corresponding to $z_{2 . i}, z_{3 . i}, z_{4 . i}$ and $z_{5 . i}$. The number of equations is unchanged, but now the elements in $z=K \Delta$ corresponding to $i \in \mathcal{I}_{k}$ are

$$
\begin{aligned}
z_{1 . i} & =D^{2} u^{i} \Delta_{x}^{i}-I^{M} \Delta_{\mu}^{i}+D u^{i} \Delta_{u}^{i}, \\
z_{2 . i} & =\Delta_{\theta}^{i}, \\
z_{3 . i} & =\Delta_{\phi}^{i}, \\
z_{4 . i} & =\Delta_{x}^{i}, \\
z_{5 . i} & =\lambda^{i} \Delta_{u}^{i}-\Delta_{\lambda}^{i} .
\end{aligned}
$$


The perturbation argument then goes by noticing that, for $i \in \mathcal{I}_{k}$, we can control $z_{1 . i}$ with $\Delta_{\mu}^{i}$ (this affects $z_{7}$, but now $\Delta_{R}$ can be moved without interfering with $z_{2 . i}$ and $z_{3 . i}$, for $i \in \mathcal{I}_{k}$ ).

For at least one $i, b_{x}^{i} \neq 0$. This individual allows one to perturb $z_{8}$. For the individuals in $\mathcal{I} \backslash \mathcal{I}_{k}$, whose elements $z_{2 . i}$ and $z_{3 . i}$ are perturbed by $\Delta_{R}$ but for whom we can use the $\Delta_{\gamma}^{i}$, the argument is exactly the same used above.

For each possible $\mathcal{I}_{k}$, an application of the transversality theorem gives a dense subset of the set of economies $\mathcal{O}$, in which it cannot be the case that $H_{k}=0$, that is it cannot be the case that $H=0$ and $b_{x}^{i}=0$ for all $i \in \mathcal{I}_{k}$. Openness of the sets follows from standard arguments. The intersection of these finitely many sets is a dense set of economies in which it cannot be the case that $H=0$.

\section{Extensions}

The convexity of the sets of sales of contracts and of the deliveries on contracts as well as the quasi-concavity of the utility functions of individuals that characterize a convex economy fail in important cases. For instance, possible non-linearities in the pricing of contracts are captured, as already argued, by a nonconvex trading set $\Phi$.

A large economy allows for competitive equilibria in non-convex environments.

The access of individuals to private technologies that allow the transformation of endowments prior to exchange also expands the scope of the model.

Individuals are $i=(t, n) \in \mathcal{I}=\mathcal{T} \times \mathcal{N}$, where $\mathcal{T}=\{1, \ldots, T\}$ is a nonempty, finite set of types, and $\mathcal{N}=\{1, \ldots\}$ is a countably infinite set of names of individuals.

A type is described by the consumption set, $\mathcal{X}^{t}$, the utility function, $u^{t}$, the endowment, $e^{t}$, and the technology, $\mathcal{Y}^{t}$, a set of pairs of bundles of commodities.

An action by an individual of type $t$, at payoffs of contracts $R$, is

$$
a^{t, n}=\left(x^{t, n}, y^{t, n}, \theta^{t, n}, \phi^{t, n}, D^{t, n} \phi^{t, n}\right),
$$

where the domain of actions of the individual at payoffs of contracts $R$ is

$$
\mathcal{A}^{t}(R)=\left\{\begin{array}{l}
x^{t, n}=y^{t, n}+\left(R \theta^{t, n}-D^{t, n} \phi^{t, n}\right) \in \mathcal{X}^{t}, \\
\left(e^{t}, y^{t, n}\right) \in \mathcal{Y}^{t}, \\
\\
\left(\phi^{i}, D^{i}\right) \in \Phi \times \mathcal{D}, \\
a^{t}: \\
\left.\phi^{t, n}, D^{t, n}\right) \in(\Phi, \mathcal{D}), \\
y_{l}^{t, n}-D_{l}^{t, n} \phi^{t, n} \geq 0, \quad l \in \tilde{\mathcal{L}}, \\
\theta^{t, n} \geq 0 .
\end{array}\right\} .
$$


At payoffs of contracts $R$, a simple distribution of actions of individuals of type $t$ is a pair $\left(\lambda^{t}, \gamma^{t}\right)$, where $\lambda^{t}=\left(\ldots, \lambda^{t, k}, \ldots\right)$ is a probability measure on a finite set, and $\gamma^{t}=\left(\ldots, a^{t, k}, \ldots\right)$ associates an action of individuals of type $t$ with every element of the support of the measure $\lambda^{t}$. For type $t$, the aggregate portfolio of contracts purchased is

$$
\theta^{t}=\mathrm{E}_{\lambda^{t}} \theta^{t, k}
$$

the aggregate portfolio of contracts sold is

$$
\phi^{t}=\mathrm{E}_{\lambda^{t}} \phi^{t, k}
$$

and the aggregate delivery on contracts is

$$
\mathrm{E}_{\lambda^{t}} D^{t, k} \phi^{t, k}
$$

Across types, a profile of simple distributions of actions is $(\lambda, \gamma)=\left(\ldots,\left(\lambda^{t}\right.\right.$, $\left.\left.\gamma^{t}\right), \ldots\right)$.

A state of the economy is $(R, \lambda, \gamma)$, payoffs of contracts and a profile of simple distributions of actions; it is feasible if

$$
r_{m} \sum_{t \in \mathcal{T}} \theta_{m}^{t}-\sum_{t \in \mathcal{T}} \mathrm{E}_{\lambda^{t}} d_{m}^{t, k} \phi_{m}^{t, k}=0, \quad m \in \mathcal{M} .
$$

A weaker feasibility condition requires that

$$
R \sum_{t \in \mathcal{T}} \theta^{t}-\sum_{t \in \mathcal{T}} \mathrm{E}_{\lambda^{t}} D^{t, k} \phi^{t, k}=0 .
$$

For economies that are not convex, additional assumptions are required to eliminate minimum wealth points.

Assumption 7. For every individual, for consumption bundles $x$ and $\hat{x}, x_{l^{*}}=0$ and $\hat{x}_{l^{*}}>0 \Rightarrow u^{i}(\hat{x})>u^{i}(x)$. For contracts $m \in \mathcal{M} \backslash\left\{m^{*}\right\}, d_{m} \in \mathcal{D}_{m} \Rightarrow d_{l^{*}, m}=$ 0 .

A consumption bundle with zero consumption of commodity $l^{*}$ yields lower utility than any bundle with positive consumption of the commodity, and contract $m^{*}$ is the only contract that effects exchanges of the commodity $l^{*}$.

The economy is

$$
\mathcal{E}=\left\{\mathcal{T}, \mathcal{L}, \tilde{\mathcal{L}}, \mathcal{M},\left(\mathcal{X}^{t}, u^{t}, e^{t}, \mathcal{Y}^{t}\right): t \in \mathcal{T},\left(\mathcal{D}_{m}, \Phi_{m}\right): m \in \mathcal{M}\right\}
$$

At payoffs of contracts purchased, $R$, and prices of contracts $q$, the budget set of an individual of type $t$ is

$$
\beta^{t}(q, R)=\left\{(x, y, \theta, \phi, D \phi) \in \mathcal{A}^{t}(R): q(\theta-\phi) \leq 0\right\} .
$$

The optimization problem of an individual of type $t$ is

$$
\begin{aligned}
\max & u^{t}(x) \\
\text { s.t } & (x, y, \theta, \phi, D \phi) \in \beta^{t}(q, R) .
\end{aligned}
$$


Definition 4. A competitive equilibrium for the large economy model is $\left(q^{*}, R^{*}\right.$, $\left.\lambda^{*}, \gamma^{*}\right)$, prices of contracts and a state of the economy, such that

1. for every type of individuals, every action, $a^{t, k *}=\left(x^{t, k *}, y^{t, k *}, \theta^{t, k *}, \phi^{t, k *}\right.$, $\left.D^{t, k *} \phi^{t, k *}\right)$, in the support of the measure $\lambda^{t *}$, is a solution to the optimization problem at prices and payoffs of contracts $\left(q^{*}, R^{*}\right)$,

2. the payoffs of contracts lie in the convex hull of the set of deliveries of contracts $^{7}: R^{*} \in$ ConD ,

3. the state of the economy $\left(R^{*}, \lambda^{*}, \gamma^{*}\right)$ is feasible, and

4. the market for contracts clears: $\sum_{t \in \mathcal{T}} \mathrm{E}_{\lambda^{t *}} \theta^{t, k}-\sum_{t \in \mathcal{T}} \mathrm{E}_{\lambda^{t *}} \phi^{t, k}=0$.

Proposition 3. In a large economy, competitive equilibria exist.

Proof: The action correspondence of an individual of type $t$ is $\alpha^{t}(q, R)$; the compensated action correspondence is $\tilde{\alpha}^{t}(q, R)$. Since the convex hull of an upper hemi-continuous correspondence is upper hemi-continuous, an argument as in the proof of proposition 1 yields a pair $\left(q^{*},\left(R^{*}, a^{\mathcal{T} *}\right)\right.$, of prices of contracts and a feasible state of the reduced economy with a representative individual for each type, such that $a^{t *} \in$ Con $\tilde{\alpha}^{t}\left(q^{*}, R^{*}\right)$, for every individual.

For $K=2 L+2 M+L M+1$, there exists a simple distribution of actions of individuals of type $t,\left(\lambda^{t *}, \gamma^{t *}\right)$, with $\lambda^{t *}=\left(\ldots, \lambda^{t, k *}, \ldots\right), \gamma^{t *}=\left(\ldots, a^{t, k *}, \ldots\right)$, such that $a^{t, k *} \in \alpha^{t}\left(q^{*}, R^{*}\right)$, for $k=1, \ldots, K$, and $a^{t *}=\mathrm{E}_{\lambda^{t *}} a^{t, k *}$.

The state of the economy $\left(R^{*}, \lambda^{*}, \gamma^{*}\right)$ is feasible: markets for commodities clear.

As in proposition $1, q_{m^{*}}^{*}>0$.

For every type of individuals, and for every element of the support of the measure $\lambda^{t *}$, the action $a^{t, k *}$ is a solution to the optimization problem at prices and payoffs of contracts $\left(q^{*}, R^{*}\right)$. If not, there exists $a \in \beta^{t}\left(q^{*}, R^{*}\right)$ with $u^{t}(x)>$ $u^{t}\left(x^{t, k *}\right)$. The only way to exchange commodity $l^{*}$ is through contract $m^{*}$ : $x_{l^{*}}=e_{l^{*}}+\theta_{m^{*}}-\phi_{m^{*}}$. If $x_{l^{*}}=0$, then, by the strong desirability of commodity $l^{*}, x_{l^{*}}^{*}=0$, which is not possible: from the endowment, the individual can reduce net sales of $m^{*}$ and still be strictly better off than at $x^{*}$, a contradiction. If $x_{l^{*}}>0$, then the individual can increase net sales of contract $m^{*}$, and find an action $\hat{a} \in \beta^{t}\left(q^{*}, R^{*}\right)$ with $u^{t}(\hat{x})>u^{t}\left(x^{t, k *}\right)$ and $q^{*}(\hat{\theta}-\hat{\phi})<0$, a contradiction.

\section{References}

1. Akerlof, G. (1970), "The market for lemons: quality uncertainty and the market mechanism," Quarterly Journal of Economics, 89, 488-500.

2. Arrow, K. J. and G. Debreu (1954), "The existence of a general equilibrium for a competitive economy," Econometrica, 22, 265-290.

7 " Con" denotes the convex hull. 
3. Citanna, A. , A. Kajii and A. Villanacci (1998), "Constrained suboptimality in incomplete markets: a general approach and two applications", Economic Theory, 11, 495-521.

4. Citanna, A. , H. Polemarchakis and M. Tirelli (2006), "The taxation of assets," Journal of Economic Theory, 126, 299-313.

5. Citanna A. and A. Villanacci (2002) "Competitive equilibrium with moral hazard in economies with multiple commodities," Journal of Mathematical Economics, 38, 117-147.

6. Cole, H. and N. Kocherlakota (2001) "Efficient allocations with hidden income and hidden storage," Review of Economic Studies, 68, 523-542.

7. Dubey, P., J. D. Geanakoplos and M. Shubik (1990), "Default and efficiency in a general equilibrium model with incomplete markets," mimeo.

8. Dubey, P., J. D. Geanakoplos and M. Shubik (2005), "Default and punishment in general equilibrium," Econometrica, 73, 1-37.

9. Dubey, P., J. D. Geanakoplos and W. Zame (1996), "Default, collateral and derivatives," mimeo.

10. Fahri, E., M. Golosov and A. Tsyvinski (2009)"A theory of liquidity and regulation of financial intermediation," Review of Economic Studies, 76, 973-992.

11. Geanakoplos, J. D., M. Magill , M. Quinzii and J. Drèze, "Generic inefficiency of stock market equilibrium when markets are incomplete," Journal of Mathematical Economics, 19, 113-151.

12. Geanakoplos, J. D. and H. M. Polemarchakis (1986), "Existence, regularity and constrained suboptimality of competitive allocations when the asset market is incomplete," in W. P. Heller, R. M. Starr and D. A. Starrett (eds), Uncertainty, Information and Communication: Essays in Honor of K. J. Arrow, vol. III, Cambridge University Press, 66-95.

13. Geanakoplos, J. D. and H. M. Polemarchakis (2008), "Pareto improving taxes," Journal of Mathematical Economics, 44, 682-696.

14. Golosov, M. and A. Tsyvinski (2007), "Optimal taxation with endogenous insurance markets," Quarterly Journal of Economics, 122, 487-534

15. Greenwald, B. and J. E. Stiglitz (1986), "Externalities and economies with incomplete information and incomplete markets," Quarterly Journal of Economics, 101, 229-264.

16. Grossman, S. J. and O. D. Hart (1983), "An analysis of the principal -agent problem," Econometrica, 51, 7-45. 
17. Grossman, S. J. and O. D. Hart (1986), "The cost and benefits of ownership: a theory of vertical and lateral integration," Journal of Political Economy, 94, 691-719.

18. Hammond, P. J. (1987), "Markets as constraints: multilateral incentive compatibility in a continuum economy," Review of Economic Studies, 54. 399-412.

19. Hammond P. J. (1989), "Efficiently regulated competition in insurance markets with adverse selection," mimeo.

20. Hart, O. D. and J. Moore (1988), "Incomplete contracts and renegotiation," Econometrica, 56, 755-785.

21. Kehoe, Timothy J., David K. Levine and Edward C. Prescott. (2002), "Lotteries, Sunspots, and Incentive Constraints." Journal of Economic Theory 107 (November): 39-69.

22. Kocherlakota, Narayana R. (1998), "The Effect of Moral Hazard on Asset Prices when Financial Markets Are Complete." Journal of Monetary Economics 41 (February): 39-56.

23. McKenzie, L. (1954), "On equilibrium in Graham's model of world trade and other competitive systems," Econometrica, 22, 147-161.

24. Panaccione, L. (2007), "Pareto optima and competitive equilibria with moral hazard and financial markets," The B.E. Journal of Theoretical Economics, 7 (Topics), article 36.

25. Prescott, E. and R. Townsend (1984), "Pareto optima and competitive equilibria with adverse selection and moral hazard," Econometrica, 52, 21-45.

26. Radner, R. (1972), "Existence of equilibrium of plans, prices and price expectations," Econometrica, 40, 289-303.

27. Rothschild, M. and J. E. Stiglitz (1976), "Equilibrium in competitive insurance markets," Quarterly Journal of Economics, 90, 629-650.

28. Spence, M. (1974), "Job market signalling," Quarterly Journal of Economics, 87, 355-374.

29. Villanacci A., L. Carosi, P. Benevieri and A. Battinelli (2002)"Differential Topology and General Equilibrium with Complete and Incomplete Markets", Kluwer Academic Publishers. 